\title{
Electrostatic Self-Assembly of Binary Nanoparticle Crystals with a Diamond-Like Lattice
}

Alexander M. Kalsin, Marcin Fialkowski, Maciej Paszewski, Stoyan K. Smoukov, Kyle J. M. Bishop, Bartosz A. Grzybowski*

Department of Chemical and Biological Engineering and The Northwestern Institute on Complex Systems; Northwestern University, 2145 Sheridan Road, Evanston, IL 60208, USA.

*To whom correspondence should be addressed. E-mail: grzybor@northwestern.edu

Self-assembly of charged, equally sized metal nanoparticles of two types (gold and silver) leads to the formation of large, sphalerite (diamond-like) crystals, in which each nanoparticle has four oppositely charged neighbors. Formation of these non-close-packed structures is a consequence of electrostatic effects specific to the nanoscale, where the thickness of the screening layer is commensurate with the dimensions of the assembling objects. Because of electrostatic stabilization of larger crystallizing particles by smaller ones, better quality crystals can be obtained from more polydisperse nanoparticle solutions.

Crystalline aggregates composed of one or more types of metallic and/or semiconductor nanoparticles (NPs) are of great interest for the development of new materials with potential applications in areas such as optoelectronics (1), high-density data storage (2), catalysis (3), and biological sensing (4). To date, methods for the crystallization of twoand three-dimensional (3D) NP superlattices have relied on the differences in the sizes of component particles and on attractive van der Waals or hard-sphere interactions acting between them. This strategy has been successful in preparing several types of lattices [such as $A B(5), A_{2}(6), A_{5}(7)$, and $\mathrm{AB}_{13}(6)$ ], but the all-attractive nature of the interparticle potentials limits its applicability to relatively few and usually (8) close-packed structures.

To overcome this limitation, we and others $(8,9)$ have focused on systems of NPs interacting via electrostatic forces; such forces provide a basis for ionic, colloidal (9) or even macroscopic (10) crystals, but - despite some promising attempts $(8,11)$ - have not been successfully exploited for controllable or predictable long-range organization of matter at the nanoscale. Here, we report electrostatic self-assembly (10) (ESA) of oppositely charged, nearly equally-sized metallic NPs of different types into large 3D crystals characterized by sphalerite (diamond-like) (12) internal packing and of overall morphologies identical to those of macroscopic diamond or sphalerite crystals (Fig. 1 to 4). Formation of these non-close packed structures results from the change in electrostatic interactions in the nanoscopic regime, where the thickness of the screening layer becomes commensurate with the dimensions of the assembling particles, and is facilitated by the presence of smaller, charged NPs in the crystallizing solutions that stabilize larger NPs by what can be termed a nanoscopic counterpart of Debye screening.

We used Ag and Au NPs coated with $\omega$-functionalized alkane thiols (13): $\mathrm{HS}\left(\mathrm{CH}_{2}\right)_{10} \mathrm{COOH}$ [MUA] and $\mathrm{HS}\left(\mathrm{CH}_{2}\right)_{11} \mathrm{NMe}_{3}{ }^{+} \mathrm{Cl}^{-}$[TMA] (Fig. 1A). These NPs were prepared according to a modified literature procedure (14) (cf. Supplementary Online Material (15)), and had average diameters of $5.1 \mathrm{~nm}$ (with dispersity, $\sigma=20 \%$ ) for Au and $4.8 \mathrm{~nm}(\sigma=30 \%)$ for Ag (Fig. 1B). We chose this pair as a model system, because the average sizes of Au NPs passivated with MUA [self-assembled monolayer (SAM) thickness $1.63 \mathrm{~nm}$ (16)] and Ag NPs covered with TMA (SAM thickness $\sim 1.9 \mathrm{~nm}$ ) had very similar overall, average sizes ( $\sim 8.36 \mathrm{~nm}$ vs. $\sim 8.6 \mathrm{~nm}$ ).

Both types of NPs were stable and unaggregated when kept in separate aqueous solutions. At the concentration used ( $2 \mathrm{mM}$ ), the $\mathrm{pH}$ of AuMUA solution was 9.7, so the NPs presented deprotonated carboxylate groups, and the ratio of NP charges was Q(AgTMA)/Q(AuMUA) $=-0.90$ (cf. Fig. $1 \mathrm{~A})$. When the solutions were mixed, the positively charged AgTMAs interacted with the negatively charged AuMUAs. As suggested by the absence of the silver plasmon band centered at $\lambda_{\max }^{\mathrm{Ag}}=424 \mathrm{~nm}$ and concomitant growth of the gold band at $\lambda_{\max }^{A u}=520 \mathrm{~nm}$ (Fig. 1C), the interaction involved close proximity of particles of the two types within small, soluble aggregates (cf. Supplementary Online Material (15)). These aggregates precipitated rapidly when the molar ratio of the NPs was near unity and the overall charge of the NPs was neutralized (Fig. 1D). 
Crystals were obtained from the NP precipitate, from which the excess of ammonium salt hindering the crystallization process was removed by washing with water. Subsequently, the precipitate was dissolved in a $1: 4 \mathrm{v} / \mathrm{v}$ mixture of water and dimethyl sulfoxide (DMSO), and crystals were grown by slow ( $12 \mathrm{hrs}$ ) evaporation of water at $70^{\circ} \mathrm{C}$ (17). This procedure yielded large numbers of regularly faceted crystals, each composed of several million NPs, and with dimensions up to $3 \mu \mathrm{m}$ in each direction (Fig. 1 and 4). When the crystals were partly dissolved in water, the ultraviolet-visible (UV-VIS) spectra showed no blue-shift of the surface plasmon resonance (SPR) of Au and an extinguished SPR band of Ag. These data suggest that (i) Ag and Au NPs in the crystals were in close proximity and (ii) that they did not amalgamate (18) during crystallization. Amalgamation was also ruled out by performing successful crystallization without heating.

The crystal structure was solved by small-angle, powder X-ray diffraction (XRD) (cf. Supplementary Online Material (15)). The XRD spectrum in Fig. 2A shows three peaks located at $2 \theta=0.801^{\circ}, 1.308^{\circ}$, and $1.539^{\circ}$. This diffraction pattern characterizes the sphalerite (or diamond, cf. Supplementary Online Material (15)) structure with the lattice constant, $a=19.08 \pm 0.53 \mathrm{~nm}$, and with peak positions corresponding to Bragg reflections on planes specified by Miller indices (111), (220), and (311), respectively. The lattice constant agrees with the value of $a \approx 18.5 \mathrm{~nm}$ based on scanning electron microscopy (SEM) measurements (Fig. 2B). Also, the interparticle distance along the body-diagonal axis calculated from XRD data is $8.27 \pm 0.26 \mathrm{~nm}$, and is close to the value of $\sim 8.48 \mathrm{~nm}$ estimated from hard-sphere radii of individual NPs (cf. Fig. 1A) and $8.5 \mathrm{~nm}$ from the SEM (Fig. 2C). Finally, both the bulk composition of the crystals as well as the identities of NPs on crystalline faces were examined via energy dispersive spectroscopy (EDS) in SEM and in transmission electron microscopy (TEM); it was found that the bulk contents of Ag and Au were approximately equal and that the arrangement of surface particles was congruent with the XRD analysis (Fig. 3).

All of these experiments indicate that NPs are arranged on a diamond lattice with each NP surrounded by four oppositely charged neighbors at the vertices of a tetrahedron (Fig. 2D). This structure is closely related to that of ZnS, except that the NP "ions" have nearly identical radii. Not surprisingly, the overall crystal morphologies - including octahedral, truncated tetrahedral, truncated and twinned octahedral, and triangular - are identical to those observed for their macroscopic diamond or sphalerite (ZnS) counterparts (Fig. 4).

Crystallization of NPs into a diamond-like structure is mediated by screened electrostatic interactions. Screening occurs because (i) the NP cores are metallic and (ii) each charged NP is surrounded by a layer of counterions. As a result, the particles interact by short-range electrostatic potentials. To show why such interactions do not lead to more closely-packed $\mathrm{NaCl}$ or $\mathrm{CsCl}$ structures that might have been expected on the basis of NP charges alone, we first note that the screening length, $\kappa_{C}^{-1}$, for the crystallized NPs is $\sim 2.7 \mathrm{~nm}$ $(19,20)$. This short distance relative to the interparticle distance means that the electrostatic energy of the crystals is well approximated by accounting only for the interactions between nearest oppositely- and like-charged NPs.

With this simplification, crystal energies (per NP) of structures, in which each NP has $n$ oppositely- and $m$ likecharged neighbors (e.g., $n=4, m=12$ for diamond, 6 and 12 for $\mathrm{NaCl}, 8$ and 6 for $\mathrm{CsCl}(21)$ ) can be written as a sum of favorable, $n E_{o p}$, and unfavorable, $m E_{\text {like }}(d)$, contributions.

Here, $E_{o p}$ and $E_{\text {like }}$ denote, respectively, the energy of two oppositely-charged NPs brought into contact, and two nearest like-charged NPs. The value of $E_{\text {like }}$ depends on the separation, $d(m)$, between the surfaces of like-charged NPs. If $d>2 \kappa^{-1}$, the electrostatic interaction is screened and $E_{\text {like }} \approx 0$; for smaller separations, $E_{\text {like }}$ increases rapidly with decreasing $d(20,22)$. In particular, for diamond structure (Fig. 4A, left) $2 \kappa^{-1} \approx d_{d}=5.3 \mathrm{~nm}$ and only $E_{o p}$ contributes effectively to the crystal energy, which is thus favorable (i.e., negative). In contrast, for $\mathrm{NaCl}$ and $\mathrm{CsCl}$ lattices (Fig 5A, right), the values of $d(m)$ are significantly smaller (3.5 and $1.3 \mathrm{~nm}$, respectively) and the like-charge repulsions offset the energetic gain compared to the diamond lattice ( $2 E_{o p}$ for $\mathrm{NaCl}$ and $4 E_{\text {op }}$ for $\mathrm{CsCl}$ ). Overall, the diamond structure has the lowest energy.

We emphasize that this effect does not scale with the size of the assembling objects. For example, with larger particles such as those recently described in (9) and (23) the characteristic separation distance between like-charged particles is much larger than the screening length, and closepacked lattices are favored. We also note briefly that theoretical models without screening but accounting for either entropic effects (24) and/or van der Waals interactions (2528) cannot justify the formation of a diamond lattice.

Progress of the crystallization process depends on the degree of monodispersity of the nanoparticles used. Surprisingly, polydispersity skewed toward smaller particles facilitated crystallization and gave rise to crystals of better quality. To understand this effect, we performed a series of experiments under identical experimental conditions (solvent and temperature) but with NPs characterized by various size distributions (Fig. 5B). When Au particles taken from the same, narrow distribution $(\sigma=20 \%)$ but functionalized with either MUA or TMA were co-crystallized, the quality of crystals was poor, and a large proportion of NPs formed amorphous aggregates (Fig. 5B, left). In contrast, when one of the distributions was significantly broader (e.g., AgTMA 
with $\sigma=45 \%$ ) than the other (like for AuMUAs we used in the model system; $\sigma=20 \%$ ), large numbers of high-quality crystals were obtained (Fig. 5B, middle). Finally, when both distributions were broad (e.g., AgTMA with $\sigma=45 \%$ and AuMUA with $\sigma=30 \%$ ) particles stayed in solution and did not crystallize at all (Fig. 5B, right). In other words, some polydispersity - but not too much - aided crystallization.

These observations can be explained qualitatively on the basis of screening of electrostatic forces acting between large NPs by smaller particles present in solution. The electrostatic interaction between two large NPs can be approximated by a screened potential (29), in which the effective screening length decreases with increasing concentration of screening charge carriers (here, small NPs) and determines the stability of dispersed nanoparticles. When large NPs are surrounded by smaller, oppositely charged ones, the effective screening length is small, and the NPs interact weakly and do not aggregate $(30,31)$. In contrast, when no small particles are present, the screening length is large, long-range attractive electrostatic forces are strong, and flocculation (32) ensues.

Thermodynamically, the presence of small NPs shifts the equilibria between dispersed (D), amorphous-aggregate (A) and crystalline (C) phases (Fig. 5C). In the absence of small particles the chemical potential of the dispersed phase, $\mu_{1}$, is - due to the electrostatic interactions - very high compared to both $\mu_{A}$ and $\mu_{C}$. In this case, the NPs either condense via flocculation or nucleate to the crystalline phase. Because the nucleation processes are less likely to occur, the condensed phase consists mostly of amorphous aggregates. Addition of small particles weakens the electrostatic interactions significantly and lowers the potential of the dispersed phase to $\mu_{2}$, which is only slightly higher than the potentials of condensed phases A and C. Here, the effective attractive forces are sufficient to overcome the energetic barrier accompanying aggregation, but are weak enough to allow the aggregates to anneal into low-energy crystals $(9,33)$. The formation of the crystalline phase occurs via the nucleation/aggregation processes (34), in which a stable nucleus is formed if its radius, $R$, is large enough, and if the gain in the bulk energy, $\Delta E_{\text {bulk }} \propto \Delta \mu_{2 C} R^{3}$, dominates over the surface energy $\Delta E_{\text {surf }} \propto \sigma R^{2}$, where $\Delta \mu_{2 C}$ and $\sigma$ denote, respectively, the difference in chemical potentials and the surface energy between dispersed and crystalline phases. The critical size, $R_{c r i t}$, of the nucleus that remains in suspension and serves as a seed for further crystallization is determined by the condition that the sum $\Delta E_{\text {bulk }}+\Delta E_{\text {surf }}-$ inversely proportional to $|\Delta \mu|$ - reaches its maximum value (34). Because $\left|\Delta \mu_{2 C}\right|<<\left|\Delta \mu_{1 C}\right|$, crystals obtained from suspension " 2 " were much larger than those formed from phase “ 1 ”. Finally, if large numbers of small particles of both types are present, the chemical potential of the dispersed phase is lower than both $\mu_{A}$ and $\mu_{C}$, and no aggregation or crystallization is observed.

Several comments are in order. We emphasize that the experimental trends cannot be explained by entropic “depletion” forces (35). In such case, the presence of small particles would destabilize the free-floating, large particles and would lead to phase separation. The electrostatic stabilization of large NPs by small ones is analogous to the Debye screening affected by high-ionic strength solutions $(36,37)$; in this respect, small, charged nanoparticles behave like ions. However, if the sizes and charges of the crystallizing particles were increased, one would need proportionally more small particles to provide efficient electrostatic stabilization (30). We have seen this effect in collections of $6 \mathrm{~nm}$ and $12 \mathrm{~nm}$ NPs we tried to co-crystallize, where the particles kept in solution could not be stabilized even by broad distributions of small NPs. Although the screening can, in principle, be modulated by increasing the ionic strength of the crystallization medium by adding salts, these salts stabilize isolated particles and also crystallize themselves - as we verified experimentally, both of these effects hinder the formation of NP crystals.

Finally, from a practical point of view, extension of the ESA approach to other types and combinations of NPs (e.g., magnetic or photoluminescent) may open new avenues to nanostructured materials of composite properties deriving from the unique properties of the diamond lattice (38).

\section{References and Notes}

1. S. A. Maier et al., Nat. Mater. 2, 229 (2003).

2. J. Hoinville et al., J. Appl. Phys. 93, 7187 (2003).

3. J. Grunes, J. Zhu, E. A. Anderson, G. A. Somorjai, J. Phys. Chem. B 106, 11463 (2002).

4. M. Zayats et al., J. Am. Chem. Soc. 125, 16006 (2003).

5. A. E. Saunders, B. A. Korgel, ChemPhysChem 6, 61 (2005).

6. F. X. Redl, K. S. Cho, C. B. Murray, S. O'Brien, Nature 423, 968 (2003).

7. A. L. Rogach, Angew. Chem.-Int. Edit. 43, 148 (2004).

8. E. V. Shevchenko, D. V. Talapin, N. A. Kotov, S. O'Brien, C. B. Murray, Nature 439, 55 (2006).

9. M. E. Leunissen et al., Nature 437, 235 (2005).

10. B. A. Grzybowski, A. Winkleman, J. A. Wiles, Y. Brumer, G. M. Whitesides, Nat. Mater. 2, 241 (2003).

11. J. Kolny, A. Kornowski, H. Weller, Nano Lett. 2, 361 (2002).

12. For crystals composed of Ag and Au nanoparticles, the lattice is isostructural with sphalerite $\mathrm{ZnS}$ (SG 216). For crystals made of only one type of metal cores (cf. Fig. 5B), the lattice is best described as diamond (SG 227). To 
account for both of these possibilities we use "diamondlike" nomenclature in the text.

13. D. Witt, R. Klajn, P. Barski, B. A. Grzybowski, Curr. Org. Chem. 8, 1763 (2004).

14. N. R. Jana, X. G. Peng, J. Am. Chem. Soc. 125, 14280 (2003).

15. Supplementary Material is available on Science Online.

16. C. V. K. Sharma, G. A. Broker, G. J. Szulczewski, R. D. Rogers, Chem. Commun., 1023 (2000).

17. After the evaporation of the "good" solvent, the NP crystals constituted only $\sim 0.01 \% \mathrm{v} / \mathrm{v}$ of the remaining solution, so crystallization cannot be attributed to confinement effects that might have been operative had all liquid been evaporated.

18. S. Link, Z. L. Wang, M. A. El-Sayed, J. Phys. Chem. B 103, 3529 (1999).

19. This estimate is based on the Deryagin-Landau-VerweyOverbeek (DLVO) theory and for a 1:1 electrolyte. Specifically, $\kappa_{C}^{-1}=\left(\varepsilon \varepsilon_{0} k_{B} T / 2 c e^{2}\right)^{1 / 2}$, where $c$ denotes the number density of ions, $e$ is the charge of an electron,

$\varepsilon$ is the static dielectric constant of the medium, and $\varepsilon_{0}$ is the permittivity of vacuum. Using energy dispersive spectroscopy, we determined the content of $\mathrm{Cl}^{-}$to be $\sim 5 \%$, which corresponds to concentration $c \sim 10^{-2} M$ of the residual ammonium salt $\left(\mathrm{NMe}_{4}^{+} \mathrm{Cl}^{-}\right)$present in the crystal. Assuming that the effective dielectric constant of a mixture of water and DMSO filling the free space between the NPs in the crystal can be approximated as $\varepsilon_{\text {eff }}=\left(\varepsilon_{\text {water }}+\varepsilon_{\text {DMSO }}\right) / 2 \approx 65, \kappa_{C}^{-1} \sim 2.7 \mathrm{~nm}$. We emphasize that this number can only be treated as an estimate, because with NPs several nanometers in diameter, we are at the limit of applicability of DLVO mean-field approach. At the same time, our approximation is qualitatively correct as verified by recent numerical Monte Carlo simulations of pairs of nanometer-sized charged particles (20).

20. J. Z. Wu, D. Bratko, H. W. Blanch, J. M. Prausnitz, J. Chem. Phys. 111, 7084 (1999).

21. C. Kittel, Introduction to solid state physics (Wiley, Hoboken, NJ, ed. 8, 2005).

22. J. N. Israelachvili, Intermolecular and surface forces (Academic Press, San Diego, ed. 2, 1992).

23. A.-P. Hynninen, M.E. Leunissen, A. van Blaaderen, M. Dijkstra, Phys. Rev. Lett. 96, 018303 (2006).

24. P. Ziherl, R. D. Kamien, J. Phys. Chem. B 105, 10147 (2001).

25. Because each NP is covered with a SAM, the only dispersion (van der Waals) interaction that needs be considered is that between alkyl chains of MUA and TMA thiols comprising the SAMs. This attractive interaction is important only if the SAMs interpenetrate; it vanishes at distances larger than $\sim 1 \mathrm{~nm}$ and its characteristic energy assuming ideal parallel alignment of two alkyl chains - is on the order of $k_{B} \mathrm{~T}$ per $\mathrm{CH}_{2}$ group $(26,27)$. TEM images of the charged NPs we used reveal that the SAMs are "rigid" (i.e., their thicknesses between aggregated NPs are the same as those on isolated ones) and that no interpenetration takes place. This observation is easily rationalized by noting that possible interpenetration of the alkyl chains would have to take place at the expense of favorable electrostatic interactions between oppositely charged head groups. Overall, the vdW forces in our system can be neglected even if two NPs touch each other. At such small separations, the interactions are dominated by attractive electrostatic forces. Finally, we note that entropic forces due to SAM squeezing (28) are also negligible, since the SAMs are not "compressible".

26. L. Salem, J. Chem. Phys. 37, 2100 (1962).

27. C.D. Bain et al., J. Am. Chem. Soc. 111, 321 (1989).

28. P. Ziherl, R.D. Kamien, Phys. Rev. Lett. 85, 3528 (2000).

29. Results of recent molecular dynamics simulations (21) indicate that mean-field screening concepts can be extended to the nanoscale, and can be used to approximate electrostatic forces acting between nanoparticles screened by counterions comparable in size (within one order of magnitude) and present in small quantities (few layers). While quantitative analogies could thus be justified, we restrict our present discussion to qualitative arguments that are sufficient to explain experimental observations.

30. V. Tohver, J. E. Smay, A. Braem, P. V. Braun, J. A. Lewis, Proc. Natl. Acad. Sci. U. S. A. 98, 8950 (2001).

31. M. Rasa, A. P. Philipse, J. D. Meeldijk, J. Colloid Interface Sci. 278, 115 (2004).

32. R. C. Ball, D. A. Weitz, T. A. Witten, F. Leyvraz, Phys. Rev. Lett. 58, 274 (1987).

33. P. C. Ohara, D. V. Leff, J. R. Heath, W. M. Gelbart, Phys. Rev. Lett. 75, 3466 (1995).

34. P. M. Chaikin, T. C. Lubensky, Principles of condensed matter physics (Cambridge University Press, Cambridge, ed. 1, 2000).

35. S. Sanyal, N. Easwar, S. Ramaswamy, A. K. Sood, Europhys. Lett. 18, 107 (1992).

36. J. Lyklema, A. d. Keizer, Fundamentals of interface and colloid science (Academic Press, London, San Diego, ed. U.S., 1995).

37. E. J. W. Verwey, J. T. G. Overbeek, Theory of the stability of lyophobic colloids (Dover Publications, Mineola, N.Y., 1999).

38. Z. Zhang, A. S. Keys, T. Chen, S. C. Glotzer, Langmuir 21, 8383 (2005).

39. S. Stoeva, K. J. Klabunde, C. M. Sorensen, I. Dragieva, J. Am. Chem. Soc. 124, 2305 (2002). 
40. We thank J.-G. Zheng and M. Kowski for helpful discussions. B.A.G. gratefully acknowledges financial support from the Camille and Henry Dreyfus New Faculty Awards Program, the National Science Foundation (Grant No. 0503673), and the ACS Petroleum Research Fund (Award \# 42953-AC5). K.B. was supported by the NSF Graduate Fellowship.

\section{Supporting Online Material}

www.sciencemag.org/cgi/content/full/1125124/DC1

Materials and Methods

Figs. S1 and S2

References and Notes

18 January 2006; accepted 8 February 2006

Published online 23 February 2006; 10.1126/science.1125124

Include this information when citing this paper.

Fig. 1. (A) Scheme and average dimensions (in $\mathrm{nm}$ ) of AuMUA and AgTMA nanoparticles used as the model system. Particle compositions estimated using the method from (39) are $\mathrm{Au}_{4100} \mathrm{~L}_{380}(\mathrm{~L}=\mathrm{MUA})$ and $\mathrm{Ag}_{3400} \mathrm{~L}_{340}(\mathrm{~L}=$ TMA); the ratio of the particles' charges $\mathrm{Q}($ AgTMA)/Q(AuMUA) = -0.90. (B) Experimental, normalized size distributions of metallic cores of $\mathrm{Ag}$ and $\mathrm{Au}$ NPs; statistics based on high-resolution TEM images of at least 500 NPs of each type. (C) Typical UV-VIS spectra for the titration of AuMUA solution (here, $2 \mathrm{mM}, 0.4 \mathrm{~mL}$ ) with small aliquots ( $40 \mu \mathrm{L}=0.1$ equiv.) of AgTMA solution (2mM; concentrations are given in moles of metal atoms). The legend gives numbers of AgTMA equivalents added. Initially, absorption of the SPR band of gold ( $\lambda_{\text {max,Au }}=520$ $\mathrm{nm})$ rises and that of the SPR band of silver $\left(\lambda_{\text {max,Ag }}=424 \mathrm{~nm}\right)$ is extinguished. Precipitation begins at $\sim 0.7$ equiv. AgTMA and is complete when [AgTMA]/[AuMUA] 0.9. The precipitation point corresponds to the formation of chargecompensated (electroneutral) complex and is consistent with an estimate derived from particle compositions:

([AgTMA]/[AuMUA] $)_{\text {neutral }}=\left(N_{A g} / N_{A u}\right) \cdot\left|Q_{\text {AuMUA }} / Q_{\text {AgTMA }}\right|=$ (3400/4100)/0.9 $=0.92$, where $N_{A g}$ and $N_{A u}$ are the numbers of $\mathrm{Ag}$ and $\mathrm{Au}$ atoms in one AgTMA and one AuMUA particle, respectively). Further addition of silver NPs solubilizes the precipitate, as evidenced by the increasing intensities of SPR bands of both types of NPs (and also by visual examination of the sample). (D) (solid curve) Progress of the titration represented by absorption coefficient $\varepsilon\left(\lambda_{\max , A u}\right)$ defined in the Supplementary Online Material (15). The initial increase in $\varepsilon$ is the result of close proximity of oppositely-charged particles within soluble aggregates (see therein). Aggregation is confirmed by the red shift of the $\mathrm{Au}$ plasmon band maximum, $\lambda_{\max , A u}$, from 520 to $\sim 558 \mathrm{~nm}$ (dashed curve). For $\chi>\sim 0.5$, precipitate redissolves, and $\lambda_{\text {max }, A u}$ decreases. (E) Large-area SEM image of binary crystals obtained from AuMUA/AgTMA precipitates.

Fig. 2. Structure of AuMUA-AgTMA binary crystals. (A) Small-angle powder XRD spectrum of the crystals. Bragg reflections on planes specified by Miller indices shown are characteristic of a diamond-like structure. Insert: Comparison between experimental $\left(d^{e}\right)$ and theoretical $\left(d^{t}\right)$ spacing between crystal planes with Miller indices $\{\mathrm{hkl}\}$. Values of $d^{t}$ were calculated based on the lattice constant $a=19.08 \mathrm{~nm}$. The center-to-center distance between nanoparticles on the (100) face, calculated as $D=\sqrt{2} a / 2$, is $D=13.49 \pm 0.37$ $\mathrm{nm}$; interparticle distance along body-diagonal axis calculated from XRD data is $8.27 \pm 0.26 \mathrm{~nm}$. (B) An SEM image of a $\{100\}_{\mathrm{SL}}$ square face taken from a twinned-octahedron crystal (insert); estimated lattice constant $a=18.5 \mathrm{~nm}$. (C) An SEM image of a $\{111\}_{\text {SL }}$ plane of a triangular face of an octahedron with estimated interparticle distance of $8.5 \mathrm{~nm}$. (D) Scheme of an AB unit cell and the projections of $\{100\}_{\mathrm{SL}}$, $\{110\}_{\text {SL }},\{111\}_{\text {SL }}$ planes. NPs of one type are positioned in the nodes of an fcc lattice, while the others occupy half of the tetrahedral voids. The crystals are isostructural with sphalerite ZnS (SG 216) or, for crystals made of only one type of metal cores (cf. Fig. 5B), with the diamond lattice (SG 227).

Fig. 3. Compositional analysis of the NP crystal faces. The picture on the left shows a typical scanning TEM (STEM) image of a hexagonal $\{111\}$ face recorded on Hitachi HF2000 instrument. The EDS analysis was performed in the STEM mode with small aperture and $2 \mathrm{~nm}$ electron probe size. The aperture settings (\#3) were chosen such as to minimize scattering from neighboring/underlying particles while retaining sufficient image contrast. Drift correction area is delineated by the red box. Graphs in (A) and (B) give typical EDS data collected along directions indicated in the STEM image at $60^{\circ}$ with respect to one another (cf. Fig. 2D). Because a measurement at each nanoparticle took $30 \mathrm{~s}$ and the residual drift of the instrument (inherent and due to sample heating) was $\sim 1 \mathrm{~nm} / \mathrm{min}$, it was possible to collect reliable data over up to four successive NPs and two scans. EDS data were obtained from the X-ray emission lines of AgL $\alpha$ (2.98 keV, blue line) and of AuL $\alpha$ (9.71 keV, magenta line). The graph in (A) corresponds to the Au-Ag-Ag-Au sequence of NPs and that in (B), to Ag-Au-Au-Ag that can be expected at these relative locations. Note that the counts are relatively high because the beam penetrates into the crystal beyond the top layer of NPs. Although X-ray emission is thus collected from several layers, signal undulation is due to the top layer - when aperture is increased (to \#1 or \#2) and signal is collected from an even larger volume/depth, the counts for Ag and Au equalize giving the $1: 1$ bulk composition of the crystal (cf. Supplementary Online Material (15)). 
Fig. 4. Different morphologies of the AuMUA-AgTMA crystals (left column) and their macroscopic sphalerite (SL) (A-D) and diamond (E) counterparts (right column): (A) octahedron, inserts show $\{111\}_{\mathrm{SL}}$ and $\{100\}_{\mathrm{SL}}$ faces; (B) cut tetrahedron, inserts show $\{111\}_{\mathrm{SL}}$ and $\{100\}_{\mathrm{SL}}$ faces from cut top and from cut edge, respectively; (C) octahedron with two triangular faces cut, insert shows the $\{111\}_{\mathrm{SL}}$ face; (D) twinned octahedron, inserts magnify $\{111\}_{\mathrm{SL}}$ faces at the location of twinning and the $\{100\}_{\mathrm{SL}}$ face of a broken neighboring crystal; (E) truncated tetrahedron, insert shows top view of the $\{111\}_{\mathrm{SL}}$ face.

Fig. 5. (A) Qualitative schemes of NP arrangements and counter-ion "atmospheres" in lattice structures considered (more realistic drawings of the lattices and the discussion of the $m$ and $n$ values defined in the main text can be found in (20)). For diamond, the separation between the like-charged particles, $d$, is larger than the sum of screening lengths, $2 \kappa_{C}^{-1}$, and the energy of repulsive electrostatic interactions is negligible. For $\mathrm{NaCl}$ and $\mathrm{CsCl}$ lattices $d<2 \kappa_{C}^{-1}$ and the repulsions between like-charged NPs offset the energetic gain of oppositely-charged interactions. (B) Effect of NP polydispersity on the quality of crystals. (a) Graphs (i) - (iii) give normalized size distributions of the metallic cores of oppositely charged NPs used in crystallization experiments; typical outcomes of these experiments are illustrated by SEM or TEM images shown in the bottom row (all scale bars correspond to $200 \mathrm{~nm}$ ). In all cases, experimental conditions were the same, and crystallization was attempted at least 5 times. (i) Co-crystallization of similarly sized AuTMA and AuMUA gave mostly amorphous aggregates. Sparse, poor quality crystals (100 to $800 \mathrm{~nm}$ ) were observed in only one out of five experiments. (ii) Crystals grown from narrowly distributed AuMUA $(\sigma=20 \%$ ) and polydisperse AgTMA ( $\sigma$ $=45 \%$, bimodal distribution with significant fraction of smaller particles of sizes 1-3 nm) were large (up to $3 \mu \mathrm{m}$ ), and regularly shaped. (iii) Broadly distributed AuMUAs ( $\sigma=$ $30 \%$ ) and AgTMAs ( $\sigma=45 \%$ ) did not aggregate or crystallize at all. (C) Effect of small particles on the stability of the dispersed, large NPs. In the absence of small particles (phase "1"), large NPs of opposite charges interact by relatively strong electrostatic forces and the dispersed phase has a high chemical potential, $\mu_{1}$. In this case, the NPs instantly flocculate to form amorphous aggregates (A). If small NPs of one type are present (phase "2"), they surround large NPs of the opposite charge and effectively screen electrostatic interactions between them. Phase " 2 " is characterized by a chemical potential, $\mu_{2}$, much lower than that of phase "1" - as a result, large NPs nucleate/aggregate into ordered crystal structures. If small NPs of both types are present in the suspension (phase " 3 "), all large NPs are screened and interact very weakly. Thus, phase " 3 " has chemical potential lower than phases A and C, and NPs remain stable in solution. 


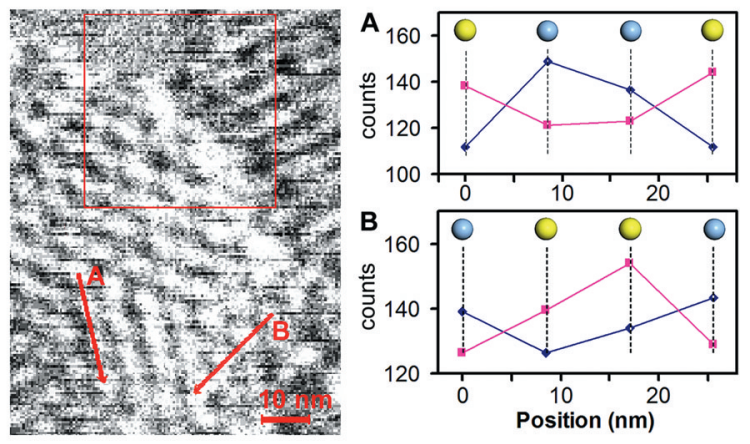




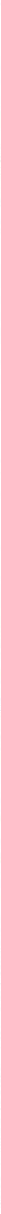



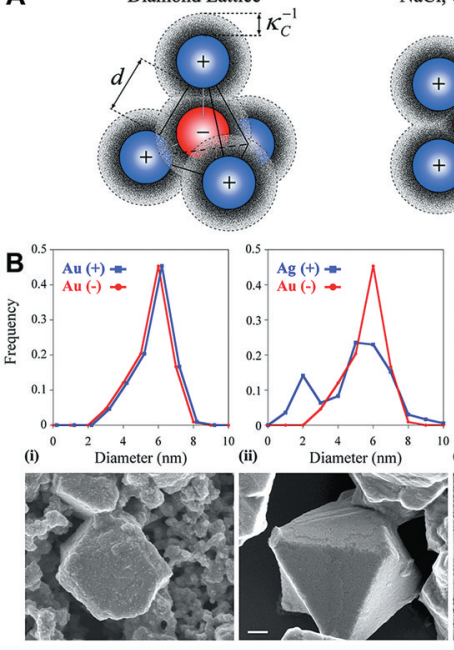

(i) Diameter (nm)

(ii)
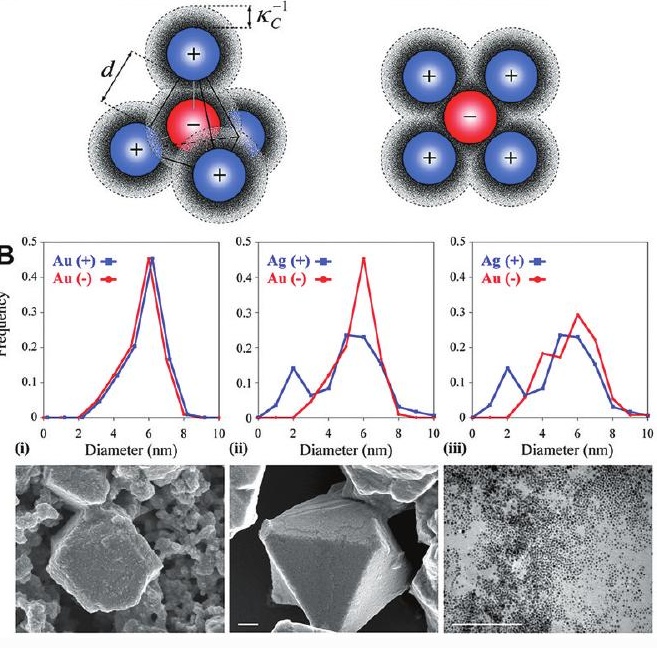

(iii) Diameter (nm)

C

flocculation

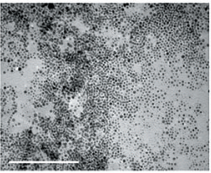

(A)

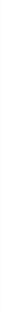

(1)

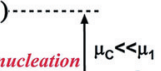

,

A 